\title{
Indices for Geometrical Properties of Cracks of RC Structures
} $\mathrm{R} \mathrm{C}$ 構造物のひび割れの幾何学的特性の指標

\author{
Tatsuya TSUBAKI* and Marian DRAGOI** \\ 椿 龍哉・マリアン ドラゴイ \\ *Member, Ph.D., Prof., Faculty of Eng., Yokohama National Univ. (Hodogaya-ku, Yokohama 240-8501) \\ ** Graduate Program, Dept. of Civil Eng., Yokohama National Univ. (Hodogaya-ku, Yokohama 240-8501)
}

\begin{abstract}
Cracks and spalling of concrete are the effects that can be seen on a reinforced concrete structure giving a warning of the occurrence of damage. An image of these effects can be taken and analyzed to estimate the damage of the structure. In this analysis image processing is necessary to extract crack data effective to characterize the damage of a particular type of structure. Characterization of geometrical properties of cracks is important in the preparation of digital crack data. In this study a set of indices to characterize the geometrical properties of cracks are examined for various RC structures.
\end{abstract}

Key Words: image processing, cracks, characterization indices, damage, reinforced concrete

\section{Introduction}

After an earthquake, damage in concrete structures appears in the form of cracking or spalling of concrete. Analyzing the crack pattern and the crack width, the damage estimate of the concrete structure can be obtained $^{1)}$. From an image of a cracked reinforced concrete structure, the digital data for crack pattern and crack width are extracted by processing the image of the damaged structure. Image processing is useful in providing necessary digital data of cracks automatically for a number of damaged concrete structures.

Once image data of cracked concrete structures are processed and the needed digital crack data are obtained, the damage analysis can be done. Thus, the first step is to get the geometrical data of cracks, e.g., by vectorization. The prototype vector data of cracks are suitable to represent the original crack pattern, while simplified vector data such as bilinear and multi-linear crack data keep the essential characteristics of the original crack pattern and are easy to handle. The simplification is based on the rule that the lines which model the actual crack must be tangent to the actual crack at the intersection points of the crack with the reinforcement so that the angle of the crack is kept the same at the position of the reinforcement. This rule is necessary because the angle between the crack and the reinforcement influences the strain value of the reinforcement.

As an application of the digitalized crack data, an estimate of the damage of the structure is considered. For the analytical modeling of a crack the smeared crack model is used, for example, being applied to four-node finite elements transforming them to orthotropic elements in the analysis. After the analysis the strain in shear reinforcement is obtained and the estimate of the maximum shear force experienced by the structure is determined by an empirical formula. Knowing the maximum shear force, the damage of the structure is estimated.

Thus, in the process of estimating the damage the digital crack data are the starting point. Image data of crack pattern are processed to obtain the indices to characterize the cracks. In the image processing pixels or dots that have the color darker than a threshold are set to represent the crack and those whiter than the threshold, the uncracked region, as follows:

$$
\begin{array}{ll}
F_{t}(I, J)=1 & (F(I, J) \geq t) \\
F_{t}(I, J)=0 & (F(I, J)<t)
\end{array}
$$

where $I, J$ indicate the position of the pixel, $t$ is the threshold intensity and $F, F_{t}$ are the intensity. In case of binary image data black and white pixels represent cracks and uncracked part, respectively.

After digitalization, indices for density, direction and distribution of cracks can be determined to characterize the geometrical properties of the crack pattern ${ }^{2), 3), 4), 5)}$.

\section{Indices by image processing}

\subsection{Index for density}

The density index is defined as the total number of black pixels on a certain area ${ }^{2), 3)}$. This index is used for a cracked concrete structure showing the total area of cracks in a specific structural zone. The total number of black pixels represents the total area of cracks. The density of cracks is an important feature of cracks. Starting from the uncracked state the density of crack shows us how cracks form and how they grow in a structural member corresponding to the load applied. Also a sudden increase of crack density without increasing the load gives the moment of failure of the structural member. The failure mode of a structural member is characterized by the shear span effective 
depth ratio $a / d$, the longitudinal and transverse reinforcement ratio, and the position and direction of reinforcement.

Density index is obtained for different areas of a structural member such as the shear span, and the span in between loading points, and the whole area of the member $^{3)}$. If the density index is large in the shear span zone, it indicates that the specimen may fail in shear. If the density index is higher at the middle of a beam or bottom of a column, possibility for flexural failure is higher. Also the reinforcement ratio and direction can be characterized from the density index. For the same specimen with different reinforcement ratio or different direction for shear reinforcement the density index is larger in case of low reinforcement ratio or smaller in case of shear reinforcement perpendicular to cracks.

\subsection{Indices for direction}

From image processing two indices for crack direction are obtained. They are the long runs emphasis ratio, LRE and the edge gradient ratio, EGR obtained from the distribution of the black pixels corresponding to cracks in a binary image data.

The LRE index is calculated by

$$
L R E=\frac{\sum_{i, j} j^{2} f(i, j)}{\sum_{i, j} f(i, j)}
$$

where $f(i, j)$ is the distribution of $i^{\text {th }}$ intensity and $j$ is the number of pixels connected in a specified direction.

In the calculation of EGR index the Kirsch operator is used. The operator is presented in Fig. 1.

\begin{tabular}{|c|c|c|c|c|c|c|c|c|c|c|c|}
\hline \multicolumn{3}{|c|}{ M0 } & \multicolumn{3}{|c|}{ M1 } & \multicolumn{3}{|c|}{ M2 } & \multicolumn{3}{|c|}{ M3 } \\
\hline 5 & 5 & 5 & 5 & 5 & -3 & 5 & -3 & -3 & -3 & -3 & -3 \\
\hline-3 & 0 & -3 & 5 & 0 & -3 & 5 & 0 & -3 & 5 & 0 & -3 \\
\hline-3 & -3 & -3 & -3 & -3 & -3 & 5 & -3 & -3 & 5 & 5 & -3 \\
\hline \multicolumn{3}{|c|}{ M4 } & \multicolumn{3}{|c|}{ M5 } & \multicolumn{3}{|c|}{ M6 } & \multicolumn{3}{|c|}{ M7 } \\
\hline-3 & -3 & -3 & -3 & -3 & -3 & -3 & -3 & 5 & -3 & 5 & 5 \\
\hline-3 & 0 & -3 & -3 & 0 & 5 & -3 & 0 & 5 & -3 & 0 & 5 \\
\hline 5 & 5 & 5 & -3 & 5 & 5 & -3 & -3 & 5 & -3 & -3 & -3 \\
\hline
\end{tabular}

Fig. 1 Kirsch operator

In Fig. 2 the values for both indices are given as a function of direction.
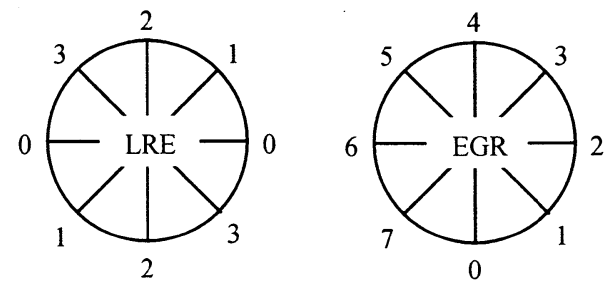

Fig. 2 Directions for LRE and EGR
The index LRE takes the values from 0 to 3 and represents the typical orientations for the runs defined for LRE: $0^{\circ}, 45^{\circ}, 90^{\circ}$ and $135^{\circ}$. In case of index EGR, values from Fig. 2 correspond to a Kirsch edge pattern. Each edge pattern represents an edge orientation or the normal direction to an edge having the direction same as index LRE.

In order to illustrate these indices two kinds of models shown in Fig. 3 are examined.

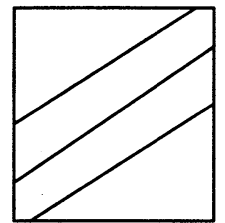

a) Model 1

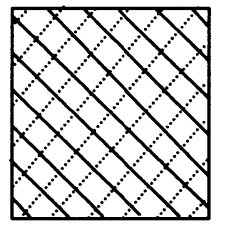

b) Model 2
Fig. 3 Model crack patterns

These models represent possible crack patterns in a concrete structure. Model 1 shown in Fig. 3 a) is a set of unidirectional cracks. Model 2 shown in Fig. 3 b) is a set of bi-directional cracks with smaller space between cracks than model 1 . Solid lines represent open cracks and dashed lines stand for closed cracks under reversed cyclic loading.

For these two models the indices LRE and EGR are calculated and presented in Fig. 4 showing the direction of cracks in terms of LRE and EGR. Analyzing the ratios LRE and EGR of Fig. 4, the principal direction of lines is determined.

Model $1 \square$ Model 2

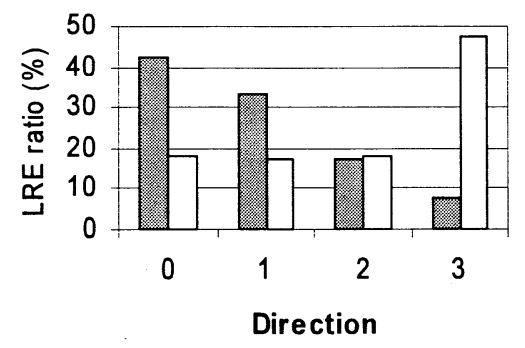

a) Index LRE

$\square$ Model $1 \square$ Model 2

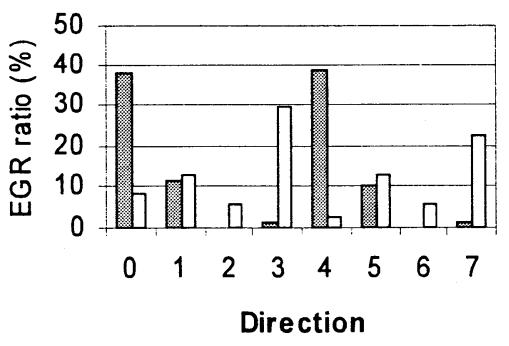

b) Index EGR

Fig. 4 LRE and EGR ratios of model crack patterns 
The LRE ratio for model 1 shows horizontal and $45^{\circ}$ principal directions, while the EGR ratio gives vertical and $135^{\circ}$ principal directions for edge orientation. The edge orientation is perpendicular to the direction of lines given by LRE. Thus, the lines of model 1 are characterized by these two indices as diagonal lines with the direction angle smaller than $45^{\circ}$. In case of model 2 , the highest ratio of the LRE ratio is direction 3 that corresponds to $135^{\circ}$. The EGR ratio shows high values for edge orientation 3 and 7 that means the direction of lines characterized by these edge patterns is perpendicular to this orientation and is $135^{\circ}$ same as the one determined by the LRE ratio.

\subsection{Index for distribution}

The distribution of the pixels of cracks for an image is characterized by the projection of all those pixels onto a line corresponding to $\mathrm{x}$ or $\mathrm{y}$ axis. Summing up all the projected dots at each position, a distribution of dots is obtained with some peak values.

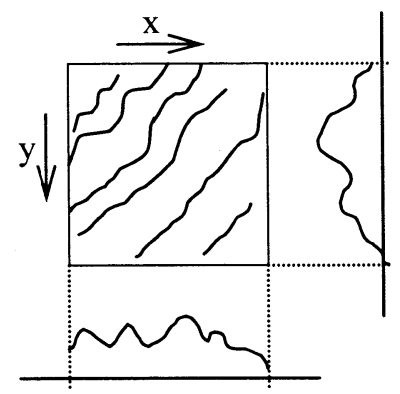

Fig. 5 Distribution of projected pixels

The representative distribution with the largest peak value is obtained by rotating the image with a specified angle increment, e.g., $5^{\circ}$ and measuring the maximum peak of each distribution. The rotation angle giving the largest peak value corresponds to the principal crack direction. From all the peaks obtained in the representative distribution only the peaks above the threshold value are considered to determine the distribution index. The distribution index consists of the distance between the peaks meaning the crack spacing. Fig. 6 shows a schematic distribution of the pixels for cracks together with the corresponding distribution index (crack spacing, $C S_{i}$ ) obtained from peak values $\left(P_{i}\right)$.

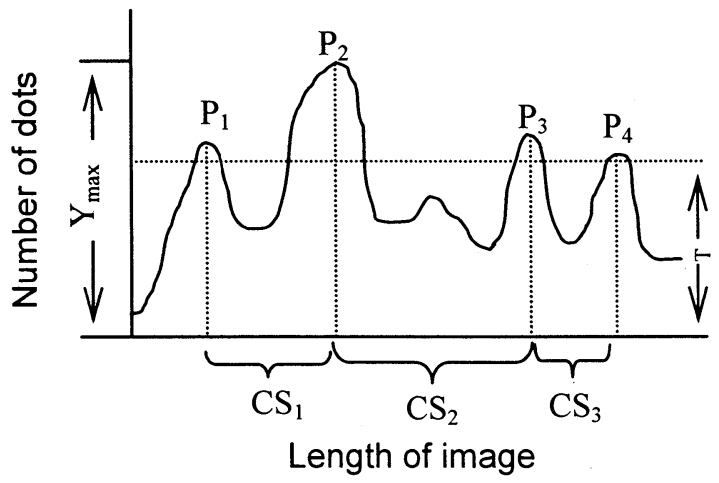

Fig. 6 Distribution index for crack spacing
Distribution index $C S$ is in accordance with the threshold value $T$ which is given by the product between maximum value of peaks, $Y_{\max }$, and a parameter $R$ that is chosen as a function of distribution of dots in the image. If cracks are not distinct and if they are close to each other, then the peak is obtained from a group of cracks.

A numerical example is given in Fig. 7 considering the same models shown in Fig. 3.

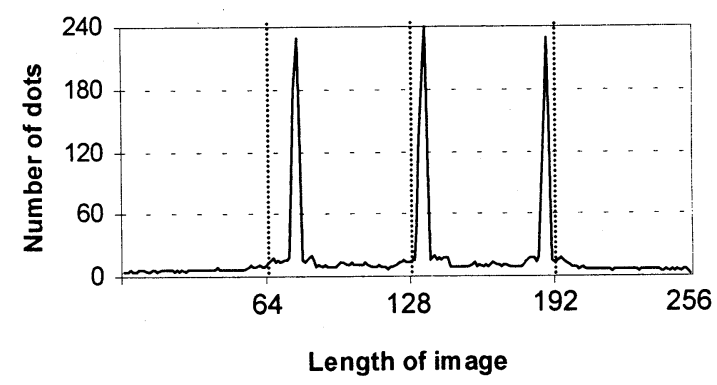

a) Model 1

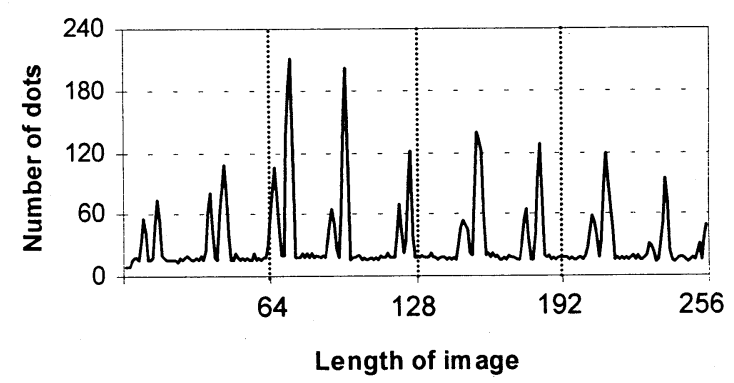

b) Model 2

Fig. 7 Distribution index of model crack patterns

In Fig. 7 the distribution index or the distance between higher peaks can be easily calculated and gives the distance between the lines of the two models. By determining the distribution index of an image representing a crack pattern, crack spacing can be determined and used in the analysis of damage estimate.

\section{Applications of indices on structural members}

Characterization of cracks in a concrete structure can be done by using the aforementioned indices obtained from image processing. Some crack patterns from different structural members such as a column, a beam and a wall are analyzed and indices from image processing are obtained to characterize the cracks.

\subsection{Column}

Crack patterns of a reinforced concrete column subjected to reversed cyclic loading ${ }^{6}$ are examined in the following. The column is of $300 \times 300 \mathrm{~mm}$ cross-section and $900 \mathrm{~mm}$ height. The longitudinal reinforcement ratio is $3.38 \%$ and the shear reinforcement ratio is $0.42 \%$. The compressive strength of concrete is $30.9 \mathrm{MPa}$. The yield load is $140 \mathrm{kN}$ and the ultimate load is $164 \mathrm{kN}$. Square images have been obtained from the shear zone near the base of the column at the beginning of loading where only flexural cracks are observed and at a stage near the 
peak load where diagonal cracks are dominant. The images of crack pattern for these two stages are shown in Fig. 8.

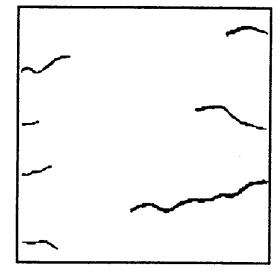

a) Flexural cracks

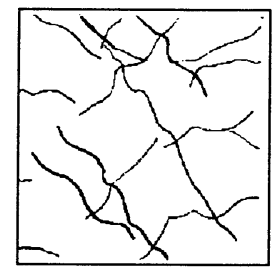

b) Shear cracks
Fig.8 Crack patterns of column for different load stages

Fig. 8 a) and Fig. 8 b) are images that have been analyzed and they correspond to the same area but different load stages. The images have been obtained from a column with high shear reinforcement ratio and no axial load.

Density index is obtained for each loading stage for the entire column and Fig. 9 shows the evolution of this index as a function of the load applied to the specimen.

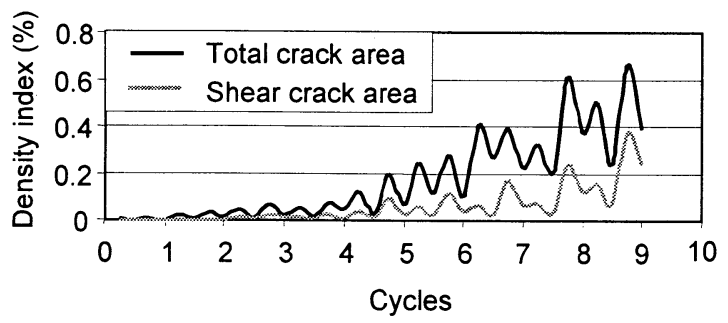

Fig. 9 Density-load relationship of column

In Fig. 9 the density index defines the evolution of crack formation considering the crack width. In early loading stages only flexural cracks are present and later, when the load increases, shear cracks appear and the density index for shear cracks starts to increase. The density index of flexural cracks is the difference between the total crack and the shear crack density indices. It is seen from Fig. 9 that in the later loading stages the density index for shear cracks increases rapidly and becomes greater than density index for flexural cracks.

For a better characterization of the cracks shown in Fig. 8 cracks are divided as follows: horizontal cracks in the left side, horizontal cracks in the right side, diagonal cracks in the left side and diagonal cracks in the right side. Thus, four images containing only cracks having one direction starting from the left side or from the right side are analyzed to obtain the indices by image processing. Indices for density are obtained from analysis and they are shown in Table $\mathbf{1 .}$

Table 1 Density index of column

\begin{tabular}{|c|c|c|c|c|}
\hline Image & HR & HL & DR & DL \\
\hline Density index (\%) & 0.8 & 0.48 & 1.79 & 2.3 \\
\hline
\end{tabular}

In Table 1, $\mathrm{H}$ comes from horizontal, $\mathrm{D}$ means diagonal and $\mathrm{R}, \mathrm{L}$ stands for the right and the left side of the column. To obtain the density index for images shown in Fig. 8, summation of the indices in Table 1 must be performed for flexural and shear cracks.

Direction indices LRE and EGR are calculated for flexural cracks from the right side and the left side of the column and they are shown in Fig. 10.

$\square$ Right $\square$ Left

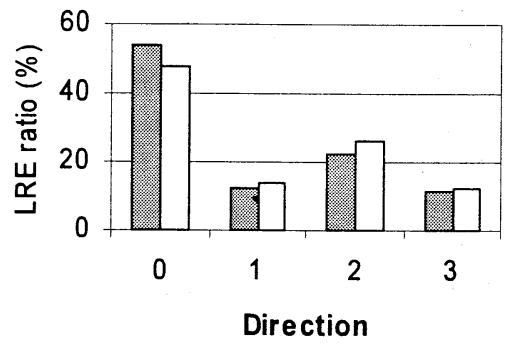

a) Index LRE

$\square$ Right $\square$ Left

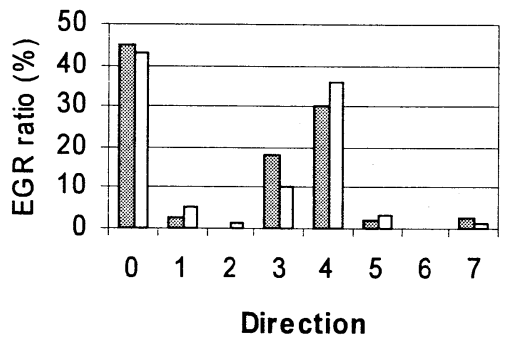

b) Index EGR

Fig. 10 LRE and EGR ratios of flexural cracks

$\square$ Right $\square$ Left

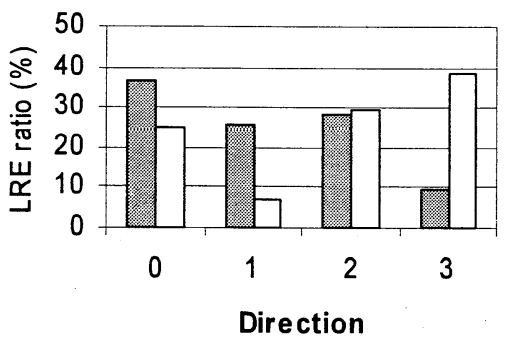

a) Index LRE

$\square$ Right $\square$ Left

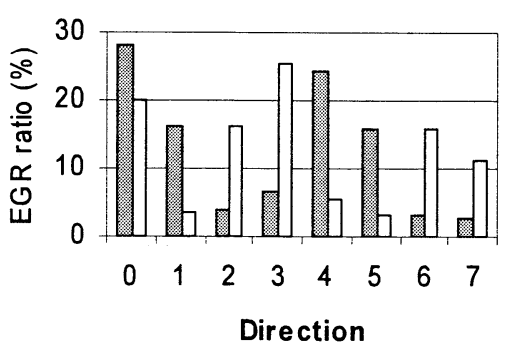

b) Index EGR

Fig. 11 LRE and EGR ratios of shear cracks 
The index LRE obtained in Fig. 10 a) shows a large ratio value for direction 0 . It means that the direction of cracks is horizontal. The edge orientation is determined in Fig. $10 \mathrm{~b}$ ) and maximum ratio values are for directions 0 and 4 that are a vertical orientation of the edge. These values confirm the principal direction of cracks given by index LRE.

Shear cracks shown in Fig. 8 are characterized by LRE and EGR indices separately, from the right side and the left side as shown in Fig. 11. In Fig.11 a) the index LRE shows a high ratio for the directions 0 and 1 for diagonal cracks from the right side. This means that the direction of shear crack is between $0^{\circ}$ and $45^{\circ}$.

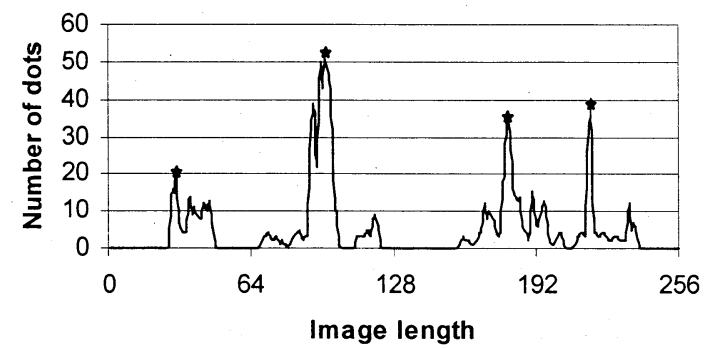

a) Distribution of dots for shear cracks from left side

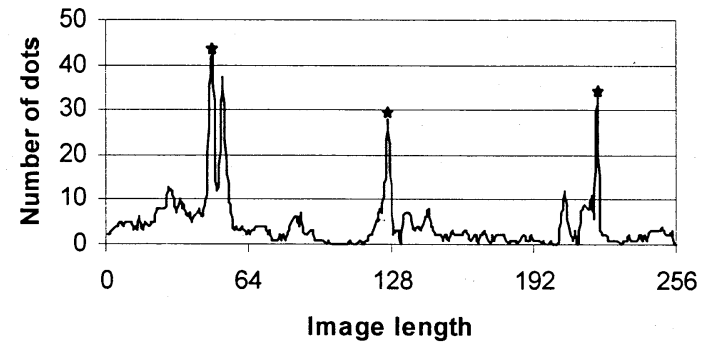

b) Distribution of dots for shear cracks from right side

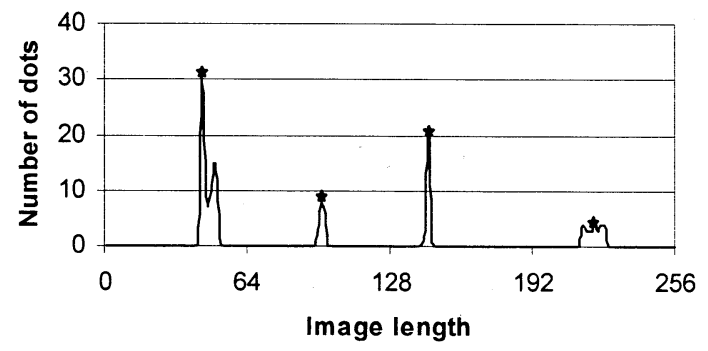

c) Distribution of dots for flexural cracks from left side

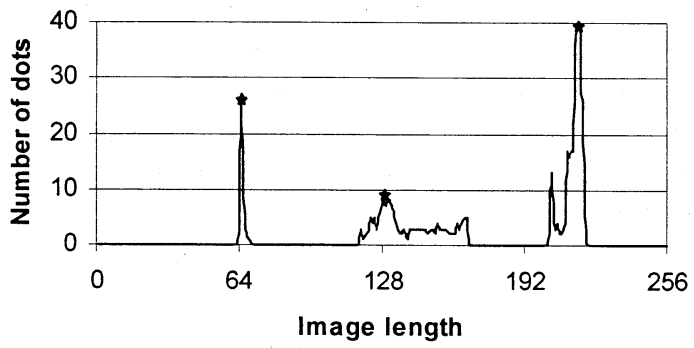

d) Distribution of dots for flexural cracks from left side

Fig. 12 Distribution index of column
The same result is obtained by index EGR showing in Fig.11 b) high values for directions $0,4,1$ and 5 of edge orientation, which is in agreement with index LRE.

For shear cracks from the left side the direction shown by index LRE is $135^{\circ}$ and the edge orientations shown by index EGR are 3 and 7 that correspond to direction given by LRE.

Thus, the directions of flexural cracks and shear cracks are well characterized by direction indices LRE and EGR obtained from images of Fig. 8.

Distribution index is also obtained from the image processing and is shown in Fig. 12 for all four cases. The distribution indices for all four cases are defined by the peak values obtained from the distribution of the crack pixels or dots which in Fig. 12 are marked by a star. The distance between these peaks gives the crack spacing for all cases. Threshold line is easily obtained because of the distinct crack patterns and for flexural cracks the threshold line is given by image length axis.

Comparing the indices obtained by image processing with the crack pattern from Fig. 8, it can be said that characterization of cracks for a reinforced concrete column by these indices is properly done.

\subsection{Beam}

Beams may have shear or flexural cracks depending on the ratio between the shear span and the effective depth, a/d. An image of crack pattern for a cracked beam tested by Suter and Manuel ${ }^{7}$ is analyzed in the following.

The beam is of a singly reinforced rectangular cross-section with $152 \mathrm{~mm}$ width, $330 \mathrm{~mm}$ height and $272 \mathrm{~mm}$ effective depth. The longitudinal reinforcement ratio is $2.44 \%$. One stirrup of \#3 rebar is placed at the position $272 \mathrm{~mm}$ away from the load point. The shear span is $544 \mathrm{~mm}$ and the shear span effective depth ratio a/d is 2.0. The load is given by two-point loading. The distance between the load and the center of beam is $203 \mathrm{~mm}$. The compressive strength of concrete is $30 \mathrm{MPa}$ and the yield strength of the longitudinal reinforcement is $460 \mathrm{MPa}$. The failure load is $180 \mathrm{kN}$. The failure mode is slow diagonal failure.

Indices for density and direction of shear cracks are found after image processing in order to characterize the cracks. In Fig. 13 the crack pattern of the beam is shown.

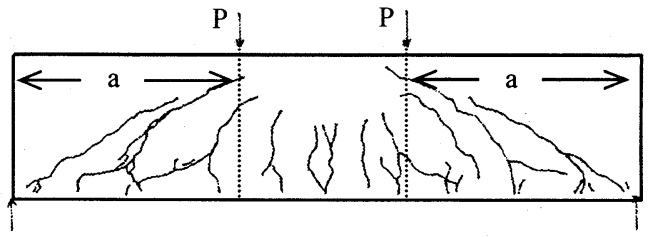

Fig. 13 Crack pattern of beam

The crack pattern is presented for a specific load stage. Up to this load stage the crack pattern was analyzed for each load stage by image processing and density index was obtained. In Fig. 14 the evolution of the density index as a function of load is shown.

Density index $D 1$ is obtained from the image that represents the area between load points (see Fig. 13). This area is representative for flexural cracks. Density 
index D2 represents the crack density in the shear span, the left and right sides of the beam.

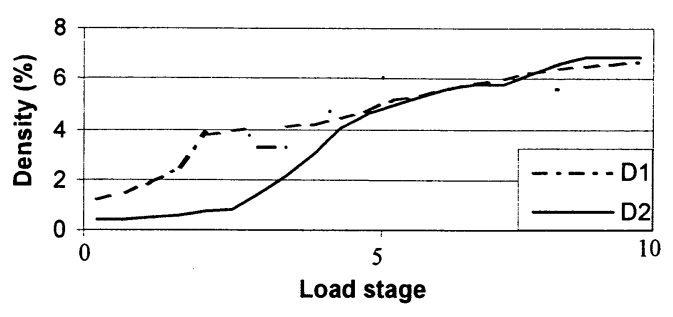

Fig. 14 Density-load relationship of beam

It can be observed in Fig. 14 that at the beginning of loading only flexural cracks are present and thus, density index $D 1$ is higher then $D 2$. As the load increases, the density of shear cracks represented by index D2 increases more rapidly than the density of flexural cracks being almost equal in the later stages. This result indicates that the development of shear cracks is slow confirming that the failure mode of this beam is slow diagonal failure. By image processing the density index is easily obtained and the density of cracks can be analyzed by using this index.

Direction of cracks for this beam is analyzed by image processing. Since in the beam there are vertical flexural cracks and diagonal shear cracks, only the area with shear cracks is analyzed to determine their direction. The image processing is done for the shear span area from the right side of the beam and direction indices LRE and EGR are obtained as in Fig 15.

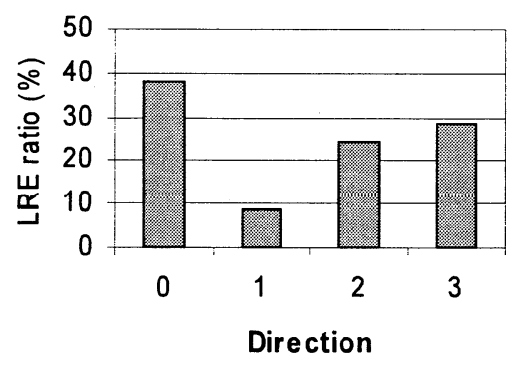

a) Index LRE

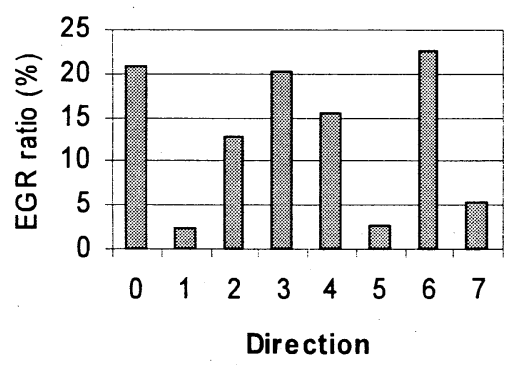

b) Index EGR

Fig. $15 \mathrm{LRE}$ and EGR ratios of beam
Direction index LRE From Fig. 15 a) and direction index EGR from Fig. 15 b) give the direction of shear crack from the image. LRE ratio is high at $0^{\circ}$ and $45^{\circ}$ and EGR ratio is high for edge orientation 0,6 and 3. By these two direction indices, cracks can be characterized as having diagonal direction at an angle of $45^{\circ}$.

\subsection{Wall}

To obtain the indices to characterize the geometrical properties of cracks in a wall, the crack pattern for the reinforced concrete wall tested by Aoyama et al.(1982) ${ }^{8)}$ is analyzed by image processing. The crack pattern of the wall is given in Fig. 16.

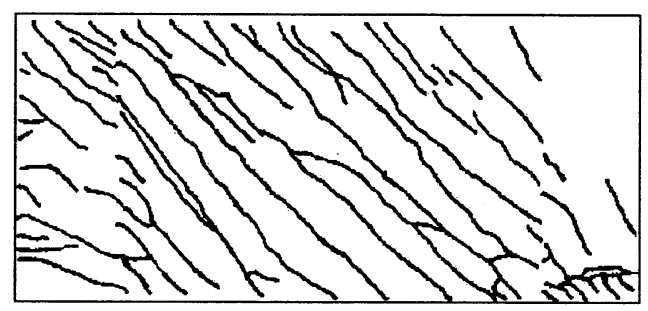

Fig. 16 Crack pattern of wall

This reinforced concrete wall is of one layer type and has an I-shape cross-section both horizontally and vertically. Non-uniform cross-sectional shape is due to the top and bottom beams and the left and right columns attached to the wall. The height and width of the wall shown in the image in Fig.16 are $970 \mathrm{~mm}$ and $2400 \mathrm{~mm}$, respectively. The shear span effective depth ratio $a / d$ is 0.55 . The wall reinforcement ratio is $0.85 \%$. The wall is subjected to cantilever type reversed cyclic horizontal loading with the load applied at the top beam and the bottom beam fixed. The left and right columns are subjected to axial compressive load of $120 \mathrm{kN}$ for each. The maximum horizontal shear force is $1650 \mathrm{kN}$.

The crack pattern shown in Fig.16 is the one at the final loading stage. It is reported that the crack width of diagonal cracks connecting the corners of the wall increased at the ultimate loading stage. The wall failed due to the compressive failure of concrete at the center of wall. This crack pattern corresponds to the one when the load is applied from the left direction. Because the applied loading is reversed cyclic, the cracks appearing at the loading in the opposite direction are closed in this condition.

It can be seen that there are mostly diagonal cracks in the wall. The characterization of the crack pattern from Fig.16 is done by the direction indices in terms of the long runs emphasis LRE index and the edge gradient ratio EGR index. By image processing indices LRE and EGR for direction of cracks are obtained and they are shown in Fig. 17.

It can be noticed in Fig. 17 a) that the direction of cracks in the wall is well characterized by index LRE having the highest ratio for direction 3 which is $135^{\circ}$. Index EGR from Fig. 17 b) confirms that the edge orientations are directions 3 and 7 that correspond to $45^{\circ}$ direction. Since the edge orientation is perpendicular to the direction of crack, the crack direction is $135^{\circ}$. 


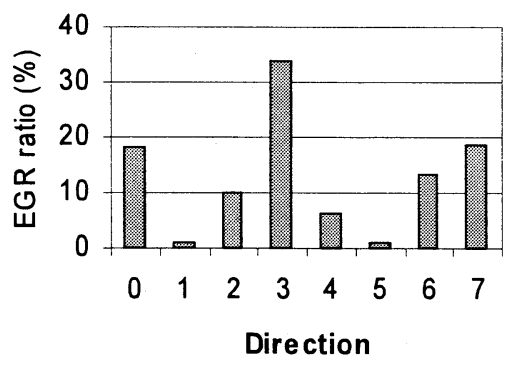

a) Index LRE

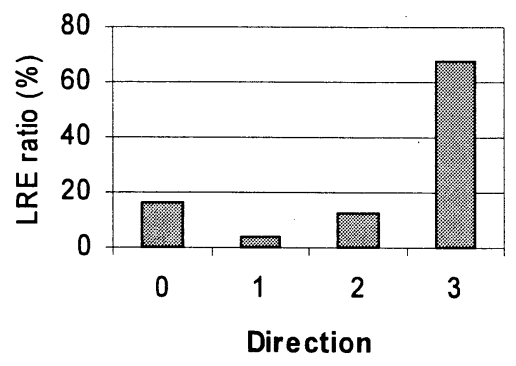

b) Index EGR

Fig. 17 LRE and EGR ratios of wall

The same direction was determined by LRE index. If the cracks in a wall follow almost the same direction, then between these two indices there is no difference and characterization of the crack direction is well made by image processing indices.

\subsection{Alkali-silica reaction in concrete}

In the concrete structures cracks appear due to alkali-silica reaction (ASR) ${ }^{9)}$ and characterization of these cracks can be made by the use of image processing indices in terms of direction and spacing. A black and white binary image is used to analyze cracks in concrete due to ASR. The resulting cracks from this reaction are not distinct and continuous as those obtained by loading effect but they follow a specific direction and show a certain crack spacing. An example of crack pattern from alkali-silica reaction ${ }^{9)}$ is presented in Fig. 18. This crack pattern is from the affected concrete beam whose age is approximately 12 years. The beam is located in the south west of the UK. Since there is little change between this crack pattern and the one observed several years before, it is said that this crack pattern is the stable one.

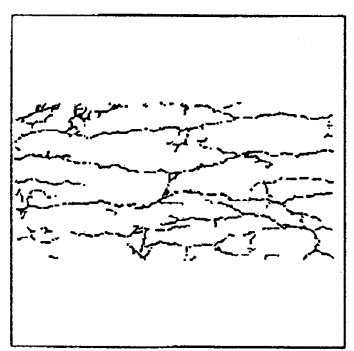

Fig. 18 Crack pattern of alkali-silica reaction

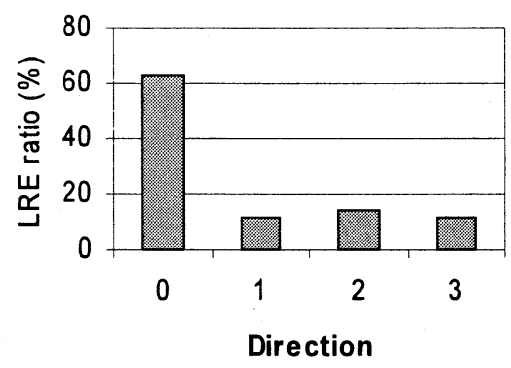

a) Index LRE

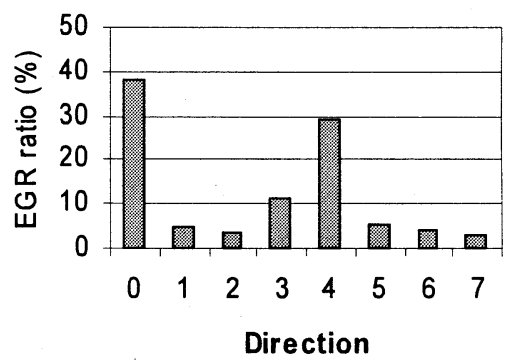

b) Index EGR

Fig. 19 LRE and EGR ratios of alkali-silica reaction

From this image, the direction of cracks due to alkali-silica reaction in the concrete beam can be characterized by indices LRE and EGR which are determined from image processing. In Fig. 19 the ratios of these indices are shown.

Index LRE gives direction 0 with the highest ratio for the principal crack direction. It means that mostly horizontal cracks are dominant in this concrete beam affected by ASR.

Index EGR has the high ratio for orientations 0 and 4 of the edge gradient. Those orientation values indicate a vertical edge orientation. If edge orientation is vertical, then the crack is horizontal, confirming the direction given by index LRE. It is confirmed that the two indices can characterize the dominant crack direction as horizontal direction.

From the image shown in Fig. 18 the crack spacing can be obtained for the horizontal direction given by distribution index as shown in Fig. 20.

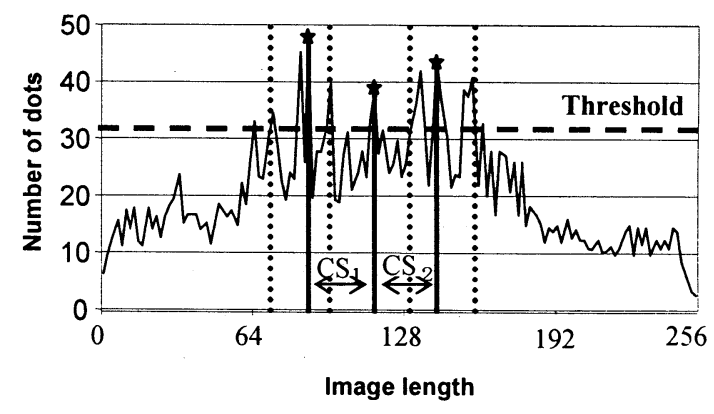

Fig. 20 Distribution index of cracks by ASR 
Because the cracks are not distinct in case of ASR, it is not easy in this case to choose the threshold value. It can be seen for the image of Fig.18 that the peaks of the number of pixels or dots projected to a line are very close to each other and have almost the same height in the middle of the image. This means that the main cracks in the middle of the image in Fig.18 are continuous from the left side to the right side of the image in the same way. Observing these peaks, the threshold value is determined so that these horizontal cracks are grouped into three major crack regions where the distribution index is given by the highest peak from each region.

Because formation of high peak groups takes place, the separation of these groups into a certain number of regions is necessary to delimit the group of cracks. The regions are delimited in Fig. 20 by dotted vertical lines and peaks corresponding to these regions are determined. It can be noticed that three main groups of cracks are obtained by delimitation and $C S$ distribution index can be determined by the position of the peaks.

Even if the cracks are not distinct in this case of a reinforced concrete beam with ASR, they can be grouped into a certain number of regions and as a result of the distribution of projected pixels or dots representing cracks, the distribution index $C S$ gives the spacing between the main group of cracks. In order to characterize the geometrical properties of cracks of concrete structures with ASR, it is necessary to recognize the two-dimensional crack pattern and the density indicating the crack width as well as the direction and spacing discussed in the above.

\section{Conclusions}

Geometrical properties of cracks are very important in the preparation of digital data by extracting the effective cracks for a given problem. To characterize the geometrical properties of cracks by image processing indices shows the potential of the image processing in the analysis of a cracked reinforced concrete structure. In the various types of structural members analyzed and discussed in this work the image processing indices such as density, direction and distribution are examined if they can characterize with accuracy the crack properties. Accuracy is obtained and improved by choosing the proper area to be analyzed. Area with both diagonal and flexural cracks must be analyzed with a method to indicate the dominant cracks by using, e.g., the information on the crack width. If the image area to be analyzed is small and only one kind of cracks appear, then indices from image processing define properly the properties of the cracks.

The results for image processing indices examined in this work are summarized as follows.

(1) The density of crack and its history can indicate how the structure failed or it will fail by analyzing the area of interest in the reinforced concrete structural members under consideration.
(2) The direction and distribution of cracks for specific structural elements give an idea about analytical modeling of crack to be used in the finite element analysis to estimate the damage. The direction index is directly connected to the type of cracks, flexural or diagonal, while the distribution index indicates the spacing between cracks.

(3) All the indices were obtained locally by the image of a specific area. In order to judge the crack pattern globally, knowledge must be accumulated and sufficient database for various structural elements must be obtained and collected to create an expert system. Such a database should contain parameters such as structural dimensions, material data, applied load conditions, load-displacement records, crack width and strains in reinforcement.

(4) In the analysis to estimate damage, in order to obtain accurate results, the proper crack data must be obtained and used together with the analytical model that is suitable for that crack data. Comparison should be made to get the simple and efficient way to implement effective crack models in the finite element analysis.

\section{REFERENCES}

1) Dragoi, M. and Tsubaki, T.: Damage estimate analysis of a reinforced concrete member subjected to reversed cycling loading, Proc. of the Sixth International Summer Symposium, JSCE, pp.337-340, 2004.

2) Tsubaki, T.: Fundamental study on crack distribution properties of concrete structures, Proc. of the $48^{\text {th }}$ Annual Conference of the JSCE, V, pp. 306-307, 1988.

3) Sakamoto, K.: Quantitative Characteristics of Crack Distribution in Concrete Structures, Graduation Thesis, Yokohama National University, 1988.

4) Masujin, K.: Directional Characteristics of Crack Distribution of RC Structures, Graduation Thesis, Yokohama National University, 1989.

5) Sakamoto, K.: Distribution Characteristics of Cracks in Concrete Structures, Master Thesis, Yokohama National University, 1991.

6) Ohtaka, M., Hayashi, K. and Tsubaki, T.: Shear crack width of RC columns subjected to reversed cyclic loading, Proc. of JCI, Vol. 26, pp. 1033-1038, 2004.

7) Suter, G.T. and Manuel, R.F.: Diagonal crack width control in short beams, ACI Journal, Vol. 68, No. 6, pp.451-455, 1971.

8) JCI: Collected Experimental Data of Specimens for Verification of Analytical Models, Proc. of JCI $2^{\text {nd }}$ Colloquium on Shear Analysis of RC Structures, JCI-C6, pp.43-54, 1983.

9) Hobbs, D.W.: Alkali-silica Reaction in Concrete, Thomas Telford, London, pp.82-86, 1988.

(Received April 15, 2005) 